\title{
过氧化氢选择性氧化硫醚的研究进展
}

\author{
刘课艳 偶辉石先莹* 董雪芬 马文娟 魏俊发*
}

(陕西师范大学化学化工学院 陕西省大分子科学重点实验室 西安 710062)

\begin{abstract}
摘要 亚砜和砜类化合物具有广谱生物活性而有广泛的应用前景，同时作为有机合成的重要中间体广泛应用于碳一碳 键形成、分子重组反应中. 硫醚直接氧化是制备亚砜和砜的主要方法之一. 在众多关于硫醚选择性氧化反应的研究中, 过氧化氢作为清洁氧化剂备受关注. 鉴于此, 就过氧化氢选择性氧化硫醚反应中的重要金属催化体系和一些非金属催 化体系的研究状况作一综述, 简要介绍各类催化氧化体系的催化效果.
\end{abstract}

关键词 催化氧化; 硫醚; 砜; 亚砜; 过氧化氢

\section{Recent Progress in the Selective Oxidation of Sulfides with Hydrogen Peroxide}

\author{
Liu, Keyan $\quad$ Ou, Hui $\quad$ Shi, Xianying* Dong, Xuefen $\quad$ Ma, Wenjuan Wei, Junfa* \\ (Key Laboratory for Macromolecular Science of Shaanxi Province, School of Chemistry and Chemical Engineering, \\ Shaanxi Normal University, Xi'an 710062)
}

\begin{abstract}
Sulfoxides and sulfones have broad applications in pharmaceutical agents and pesticide due to their biologically activities and they are important synthetic intermediates in $\mathrm{C}-\mathrm{C}$ bond formation and various molecular rearrangements. The selective oxidation of sulfides is a straightforward and frequently used method to obtain sulfoxides and sulfones. Among various oxidizing agents, hydrogen peroxide as benign oxidant has attracted organic chemists' attention for a long time. This review summarizes the recent and important catalytic systems of metal and nonmetal in this area. The catalytic effect of different catalytic systems is briefly discussed herein.
\end{abstract}

Keywords catalytic oxidation; sulfides; sulfones; sulfoxides; hydrogen peroxide

亚砜和砜类化合物具有广谱生物活性而有广泛的 应用价值, 同时作为有机合成的重要中间体广泛用于 碳一碳键形成、分子重组反应中. 硫梄直接氧化是制备亚 砜和砜的主要方法之一, 实现这一氧化反应常用的氧化 剂包括金属氧化物、无机和有机氧化剂等. 这些氧化剂 在不同程度存在着缺点, 例如一些氧化剂在反应过程中 产生等量的低价态还原产物, 这不但增加了产物分离提 纯的难度, 而且处理这些废弃物时, 会对环境产生不良 影响.

在普遍提倡环境保护、绿色生产、讲究原子经济性 的今天, 用绿色氧化剂, 例如: 氧气、臭氧、固定化的氧 化物、生物氧化酶、过氧化氢等 ${ }^{[1]}$, 代替传统的氧化剂
是实现清洁生产的关键. 其中, 过氧化氢因价廉、反应 后处理简单、副产物为水, 作为绿色氧化剂受到化学工 作者的特别关注. 但过氧化氢的缺点在于它是一种具有 中等氧化能力的氧化物, 因此, 以过氧化氢作为氧化剂 实现绿色氧化的主要问题是建立高选择性、高效的催化 体系.

近年来关于过氧化氢选择性氧化硫醚的研究, 尤其 是在催化剂的制备和篎选方面, 得到了快速的发展. 文 献中报道了不同类型催化剂催化过氧化氢氧化硫醚的 反应. 本文就硫醚氧化反应中的重要金属催化体系和一 些非金属催化体系的研究状况作一综述, 简要介绍各类 催化氧化体系的催化效果.

\footnotetext{
*E-mail: shixy@snnu.edu.cn; weijf@snnu.edu.cn

Received October 16, 2013; revised November 25, 2013; published online December 18, 2013.

Project supported by the National Natural Science Foundation of China (Nos. 21072123, 21272145), the Fundamental Research Funds for the Central Universities (Nos. GK201102005, GK261001095).

国家自然科学基金(Nos. 21072123, 21272145)、中央高校基本科研业务费专项资金(Nos. GK201102005, GK261001095)资助项目.
} 


\section{1 铇催化体系}

以往的研究已证明铇催化剂是催化过氧化氢氧化 反应的有效催化剂 ${ }^{[2]} . \mathrm{Na}_{2} \mathrm{WO}_{4}$ 、过氧铇配合物和相转移 催化剂同时使用，被应用于硫醚的氧化反应 ${ }^{[3]}$. Sato 等 ${ }^{[4]}$ 在均相体系中研究了 $\left[\mathrm{CH}_{3}\left(n-\mathrm{C}_{8} \mathrm{H}_{17}\right)_{3} \mathrm{~N}\right] \mathrm{HSO}_{4}$ 为相转移催 化剂, $\mathrm{C}_{6} \mathrm{H}_{5} \mathrm{PO}_{3} \mathrm{H}$ 调节 $\mathrm{pH}$ 值, $\mathrm{Na}_{2} \mathrm{WO}_{4}$ 催化过氧化氢氧化 硫醚. 实验结果表明采用 2.5 equiv. 的过氧化氢, 无溶剂 条件下，二烷基硫醚、烷基芳基硫醚都可以被高产率 $(87 \% \sim 98 \%)$ 的氧化为砜.

2009 年, Gharah 等 ${ }^{[5]}$ 合成了两种钼铇的过氧配合物: $\mathrm{PPh}_{4}\left[\mathrm{MoO}\left(\mathrm{O}_{2}\right)_{2}(\mathrm{HPEOH})\right]$ 和 $\mathrm{PPh}_{4}\left[\mathrm{WO}\left(\mathrm{O}_{2}\right)_{2}(\mathrm{HPEOH})\right]$. 乙腈溶剂、短时间内, 可以将硫醚氧化为亚砜或砜类物 质(Eq. 1). 虽然催化剂用量仅为 $0.1 \mathrm{~mol} \%$, 亚砜或者砜 的产率 $\geqslant 94 \%$, 但过氧化氢用量为硫醚的 4 6 倍, 而且 反应需在回流条件下进行.

Sulfides $\frac{\mathrm{PPh}_{4}\left[\mathrm{WO}\left(\mathrm{O}_{2}\right)_{2}(\mathrm{HPEOH})\right](0.1 \mathrm{~mol} \%)}{\mathrm{CH}_{3} \mathrm{CN}, \mathrm{H}_{2} \mathrm{O}_{2}(4 \sim 6 \text { equiv. }), \text { reflux }}$ Products

2012 年, Xue 等 ${ }^{[6]}$ 合成了一种温度响应的相转移催 化剂 $\left[\left(\mathrm{C}_{18} \mathrm{H}_{37}\right)_{2}\left(\mathrm{CH}_{3}\right)_{2} \mathrm{~N}\right]_{7} \mathrm{PW}_{11} \mathrm{O}_{39}$, 并将其应用于催化过 氧化氢氧化硫醚的反应(Scheme 1), $60{ }^{\circ} \mathrm{C} 、 1,4$-二氧六环 溶剂中, 研究了此催化剂的催化活性和选择性. 结果显 示, 反应 $0.5 \mathrm{~h}$ 后各种硫醚以大于 $96 \%$ 的转化率氧化为 相应的砜, 且砜的选择性都达到 99\%. 反应后催化剂可 以从反应体系中沉淀出来循环使用, 但循环使用 6 次后 转化率降到 $76 \%$.

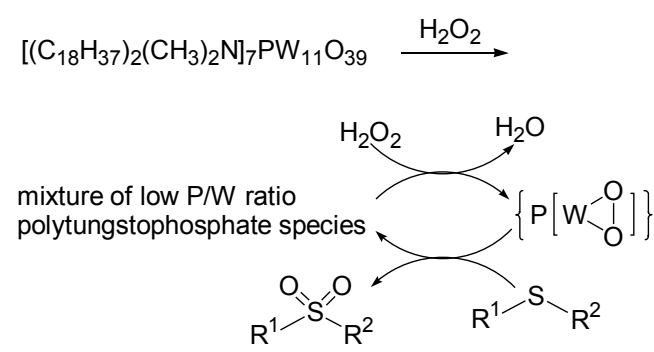

\section{Scheme 1}

2012 年, Das 等 ${ }^{[7]}$ 以三种单核、双核过氧铇酸为催 化剂, 室温下, 甲醇与水混合溶剂中, 实现了选择性催 化氧化硫醚为亚砜或砜的反应. 亚砜和砜的产率均超过 $93 \%$, 特别是活性较差的苯并塞吩也可在 $65{ }^{\circ} \mathrm{C}$ 时以 97\%的转化率得到氧化产物. 三种过氧铇酸催化剂的活 性没有明显的差别. 此外, 他们对反应的机理进行了描 述: 亲电性的氧原子从双过氧铇配合物转移到硫醚, 给 出氧化产物亚砜和去活性的单过氧铇中间体, 后者在过 氧化氢存在下形成活性双过氧铇配合物, 重新开始催化 循环，亚砜也可以继续被氧化为砜(Scheme 2).

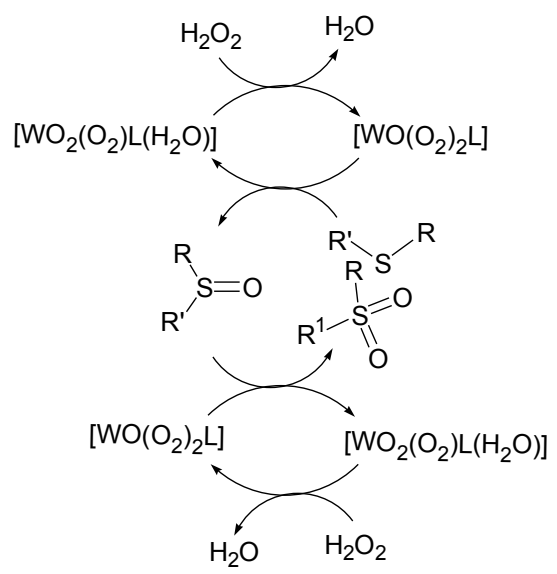

Scheme 2

固载铇催化剂在硫醚氧化反应中也有广泛的应用. 2005 年, $\mathrm{Koo}$ 等 ${ }^{[8]}$ 将 $\mathrm{WO}_{3}$ 固载于分子篮 $\mathrm{MCM}-48$ 上, 甲 醇溶液中催化过氧化氢氧化硫醚. 当过氧化氢为底物的 1.1 倍，室温下反应 $4 \mathrm{~h}$ ，硫醚被选择性氧化为相应的亚 砜，产率超过 $90 \%$; 当过氧化氢为底物的 3 倍, $60{ }^{\circ} \mathrm{C}$ 反 应 $12 \mathrm{~h}$ ，硫醚被选择性地氧化为砜，产率超过 95\%. 此 固载催化剂虽然催化活性高, 但需要浓度为 $50 \%$ 的过氧 化氢, 而且完成反应需要较长的时间.

2005 年, Karimi 等 ${ }^{[9]}$ 通过简单的方法将铇酸根阴离 子载于硅胶表面, 催化过氧化氢氧化硫醚为亚砜或砜 (Eq. 2). 虽然催化剂重复使用 8 次, 产物甲基苯基亚砜 的产率基本没有下降，但各种硫醚氧化为相应亚砜的产 率最高仅为 $91 \%$, 而且此催化体系需要 $3 \sim 8$ 倍量的过 氧化氢.

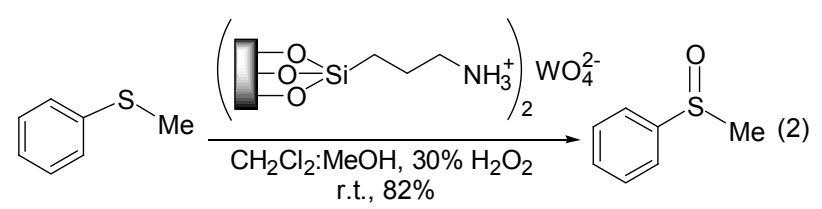

2011 年, 我们研究小组 ${ }^{[10]}$ 以二氧化硅为载体, 合成 了一种新型的双层离子液体刷固载过氧磷铇酸盐催化 剂, 考察了催化剂催化 30\%过氧化氢溶液选择性氧化硫 醚为亚砜或砜的催化性能(Eq. 3). 该催化剂具有很高的 催化活性和选择性. 采用 1.1 倍 $\mathrm{H}_{2} \mathrm{O}_{2}$ 时, 反应选择性地 生成亚砜(产率 $83 \%$ ～96\%), 仅有微量砜形成; 采用 2.5 倍 $\mathrm{H}_{2} \mathrm{O}_{2}$ 时，反应选择地生成砜(产率 87\% 99\%). 重要 的是，催化氧化体系对于含有烯键的硫醚也具有很好的 化学选择性, 即使当 $\mathrm{H}_{2} \mathrm{O}_{2}$ 的用量为底物的 2.5 倍时, 也 没有环氧化产物产生. 催化剂循环使用 8 次后，甲基苯 基亚砜的产率为 $92 \%$.

2012 年, 我们 ${ }^{[11]}$ 以固载双层离子液体刷/双核过氧 铇酸盐为催化剂, 实现了纯水介质中, 催化 $30 \%$ 过氧化 氢选择性氧化硫醚为亚砜和砜的反应(Scheme 3). 纯水 

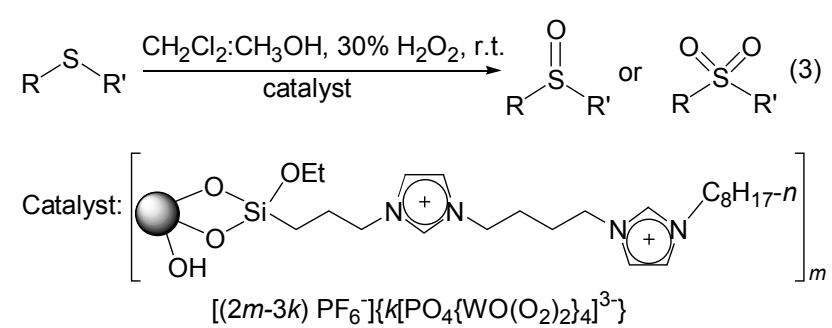

溶剂中, 多相催化剂依然表现出良好的催化活性, 其中 亚砜和砜的产率分别为 $71 \% \sim 94 \%$ 和 $86 \% \sim 100 \%$. 催 化剂循环使用 8 次后，甲基苯基亚砜产率仍然可达 $89 \%$.

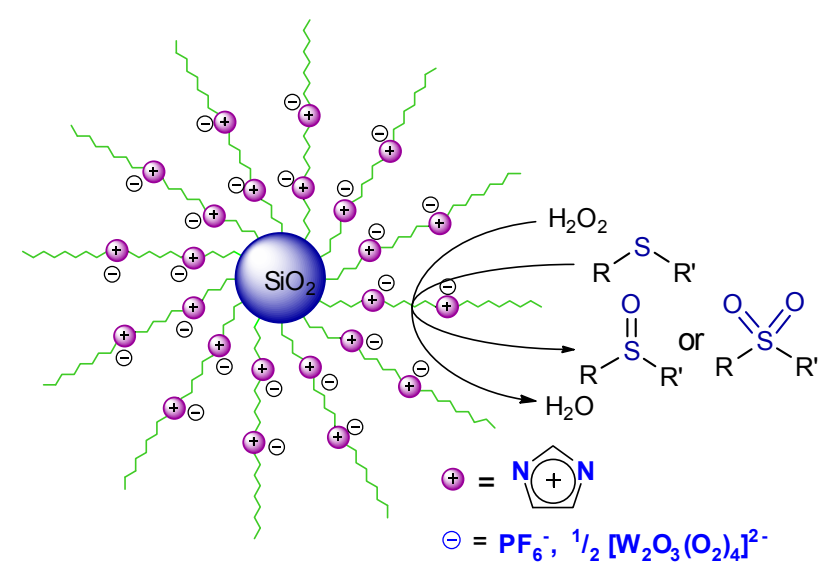

Scheme 3

\section{2 钼催化剂体系}

钿催化剂在过氧化氢的氧化反应中也表现出良好 的催化活性. 2009 年, Jeyakumar 等 ${ }^{[12]}$ 报道了七钿酸铵催 化过氧化氢氧化硫醚为砜的反应(Eq. 4). 尽管在 $1 \mathrm{~h}$ 内, 砜的产率均高于 $89 \%$, 而且对氧化剂敏感的官能团, 如 差基、双键、参键, 均不受影响. 但是催化剂和过氧化 氢的用量较大, 分别为底物的 $10 \mathrm{~mol} \%$ 和 4 倍. 同时需 要大量的有机溶剂, 底物为 $4 \mathrm{mmol}$ 时, 溶剂甲醇需要 $10 \mathrm{~mL}$.

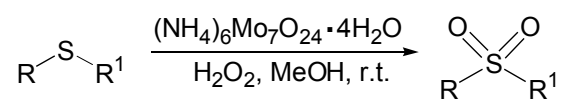

2010 年, Tundo 等 ${ }^{[13]}$ 利用 Keggin-type 杂多酸 $\mathrm{H}_{3} \mathrm{P}$ $\mathrm{Mo}_{12} \mathrm{O}_{40}$ 和 $\mathrm{Py}_{3} \mathrm{PMo}_{12} \mathrm{O}_{40}$ 在乙腈中选择性催化氧化硫醚. 室温下，几种硫醚可被高产率的氧化为亚砜，即使空间 位阻很大的二苯基硫醚, 在 $\mathrm{Py}_{3} \mathrm{PMo}_{12} \mathrm{O}_{40}$ 催化下, 也可 以得到 100\%产率的二苯基亚砜. 升高温度至 $70{ }^{\circ} \mathrm{C}, 30$ min 内, 可以将硫醚选择性氧化为砜，产率为 $97 \%$ $100 \%$. 此催化氧化体系的不足之处在于过氧化氢的用 量很大, 为底物的 20 倍.

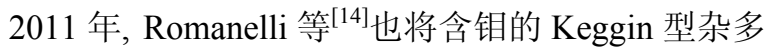
酸化合物应用于硫醚的氧化反应. 他们在 Tundo 小组的
研究基础上，合成了 4 种 Keggin 型杂多酸化合物的酸性 吡啶盐: $\mathrm{H}_{4} \mathrm{PMo}_{11} \mathrm{VO}_{40}, \mathrm{H}_{5} \mathrm{PMo}_{10} \mathrm{~V}_{2} \mathrm{O}_{40}, \mathrm{H}_{9} \mathrm{PMo}_{6} \mathrm{~V}_{6} \mathrm{O}_{40}$ 和 $\mathrm{H}_{4} \mathrm{PMo}_{11} \mathrm{VO}_{40}$. 结果表明: 在 $20{ }^{\circ} \mathrm{C}$ 或 $40{ }^{\circ} \mathrm{C}$, 乙腈为溶 剂, 四种催化剂都表现出良好的催化活性和一定的选择 性, 其中 $\mathrm{H}_{4} \mathrm{PMo}_{11} \mathrm{VO}_{40}$ 酸性吡定盐的催化活性最高. 虽 然在反应中催化剂用量仅为底物的 $1 \mathrm{~mol} \%$, 但过氧化 氢用量大, 氧化为亚砜和砜时, 过氧化氢的用量分别是 硫醚的 2.85 倍和 28.5 倍.

固载钿催化剂也被应用于硫醚的氧化反应中. 1997 年, Raghavan 等 ${ }^{[15]}$ 用水热法合成了 Mo-silicalite-1 (MoSi-1)分子篮催化剂，此催化剂没有 $\mathrm{MoO}_{3}$ 等杂质物 存在, 且 Mo 的负载量大. 甲醇溶液中, 各类型硫醚的 转化率为 $80 \% \sim 98 \%$, 亚砜的选择性为 $75 \% \sim 94 \%$. 几 种底物中, 二苯基硫醚的反应性最差, $8 \mathrm{~h}$ 后, 二苯基亚 砜的选择性仅为 $75 \%$. 尽管 $\mathrm{MoSi}-1 / \mathrm{H}_{2} \mathrm{O}_{2}$ 体系有良好的 催化氧化活性, 但需要质量分数为 $10 \%$ 的催化剂, 而且 催化剂制备时还需高温煅烧. 氧化反应可能按照如下历 程完成(Scheme 4).

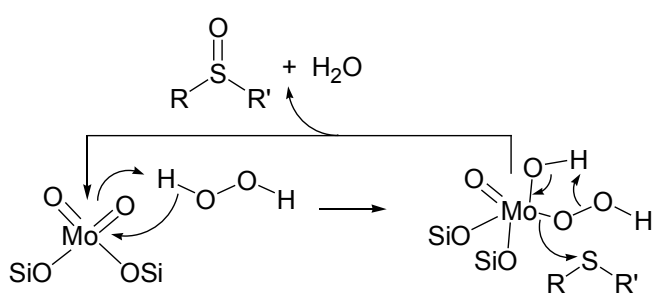

Scheme 4

2012 年, Sharma 等 ${ }^{[16]}$ 通过简单的方法将 2,6-二乙酰 吡啶邻着茎甲酰腙钼配合物载到硅胶表面, 合成了催化 剂Mo-DAPSH@APTES@ $\mathrm{SiO}_{2}$ (Scheme 5). 室温无溶剂 条件下, 催化剂催化过氧化氢氧化硫醚为亚砜的转化率 为 $82 \% \sim 100 \%$, 选择性为 $90 \% \sim 98 \%$. 相同条件下增加 过氧化氢的量到 3.6 倍时, 硫醚氧化到相应砜的转化率 和选择性都可以达到 $100 \%$. 而且此催化剂重复使用 6 次时，二苯基硫醚氧化为亚砜和砜的转化率分别为 $96 \%$ 和 $98 \%$, 选择性分别为 $94 \%$ 和 $97 \%$.

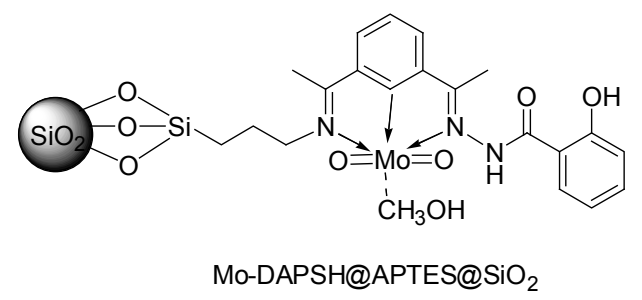

Scheme 5

2012 年, Grivani 等 ${ }^{[17]}$ 以亚胺、胺作为连接基, 将 $\mathrm{MoO}_{2} \mathrm{Cl}_{2}$ 通过配位的方式载于聚苯乙烯上得到一系列催 
化剂. 在多种脂肪族和芳香族硫醚氧化为亚砜的反应 中, 催化剂表现出很好的活性. 短时间内、纯水介质中, 利用以下催化剂(Scheme 6), 各种硫醚的转化率均达到 100\%. 以甲基苯基硫醚为底物, 该催化剂重复使用 15 次后, 硫醚的转化率依然保持在 $100 \%$. 但 Grivani 等没 有对产物亚砜的选择性进行定量.

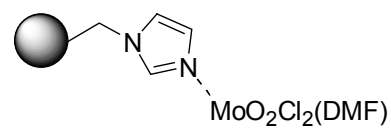

\section{Scheme 6}

\section{3 钒催化剂体系}

近几年, 钒化合物因其价格低廉、配位性能好的特 点吸引了很多化学工作者的关注 ${ }^{[18]}$. 例如 $\mathrm{NH}_{4} \mathrm{VO}_{3}$, $\mathrm{NaVO}_{3},\left(\mathrm{CH}_{3} \mathrm{COCH}_{2} \mathrm{COCH}_{2}\right)_{2} \mathrm{VO}$ 等已经广泛地应用于 催化氧化反应 ${ }^{[19]} .50 \sim 60{ }^{\circ} \mathrm{C}$, 乙腈为溶剂, Berrocal 等 ${ }^{[20]}$ 比较了 $\left(\mathrm{C}_{2} \mathrm{~N}_{2} \mathrm{H}_{10}\right)_{0.5}\left[(\mathrm{VO})\left(\mathrm{HAsO}_{4}\right) \mathrm{F}\right]$ (EnVAs) 和 $\left(\mathrm{C}_{4} \mathrm{~N}_{2}-\right.$ $\left.\mathrm{H}_{12}\right)_{0.5}\left[(\mathrm{VO})\left(\mathrm{HAsO}_{4}\right) \mathrm{F}\right]$ (PipVAs)催化氧化硫醚为亚砜的 效率. 结果表明, EnVAs 的活性较好, 底物的转化率、产 物的选择性均高于使用催化剂 PipVAs, 而且反应的 TOF 值也很高.

2012 年, Hussain 等 $^{[21]}$ 合成了一种结构新颖的钒催 化剂 $\left[\mathrm{VO}_{2} \mathrm{~F}(\mathrm{dmpz})_{2}\right](\mathrm{dmpz}=3,5$-二甲基吡唑), 仅使用 1 $\mathrm{mol} \%$ 催化剂就可以催化过氧化氢得到较高产率的亚砜 (Eq. 5). 其中 DMSO 的产率可达到 99\%, 难于被氧化的 二苯并噻吩类化合物的氧化产率也在 $75 \%$ ～ $88 \%$. 催化 剂可重复使用, 循环 5 次后甲基苯基亚砜的产率达到 86\%. 尤为重要的是用 $5 \mathrm{~g}$ 甲基苯基硫醚进行反应时, 亚砜的产率仍高达 99\%. 但此催化体系对于苯环上连有 硝基的硫醚氧化效果不好，4-硝基苯基甲基亚砜的产率 只有 $35 \%$.

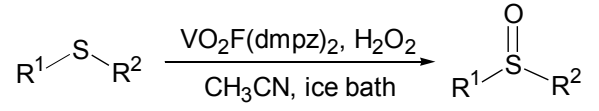

钒的一些化合物被载于载体上所形成的催化剂也 表现出优良的催化活性. 2006 年 Sullivan 等 ${ }^{[22]}$ 将钒载于 烷基磷酸修饰的硅胶上, 室温、乙腈中, 催化 2 equiv.的 过氧化氢，仅需要 $1 \mathrm{~h}, 7$ 种亚砜的选择性都达到 $99 \%$, 而且催化剂在甲基苯基硫醚的反应中可以重复使用 3 次. 但是不足的是底物的转化率没有给出.

2007 年, Prasanth 等 ${ }^{[23]}$ 将乙酰丙酮氧化钒载到强酸 性磺酸离子交换树脂上, 得到可循环使用的钒催化剂 (Scheme 7), 室温下实现了硫醚的氧化, 短时间内亚砜 的产率可以达到 $80 \%$ 以上. 催化剂循环使用 5 次, 催化 活性和选择性基本没有降低.

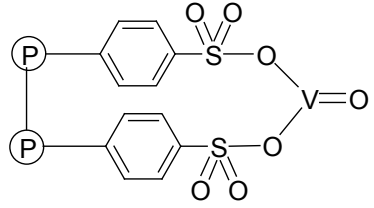

Scheme 7

2012 年, Islam 小组 ${ }^{[24]}$ 将过氧钒配合物载于高分子 上(Scheme 8), 室温下、纯水介质中, 固载催化剂可以将 8 种硫醚催化氧化为亚砜. 催化剂经简单处理即可重复 使用，在循环使用过程中，活性保持不变. 值得一提的 是，在所有底物中，反应活性不高的二苯基硫醚的转化 率和产物的选择性均最高，分别为 $95 \%$ 和 $92 \%$.

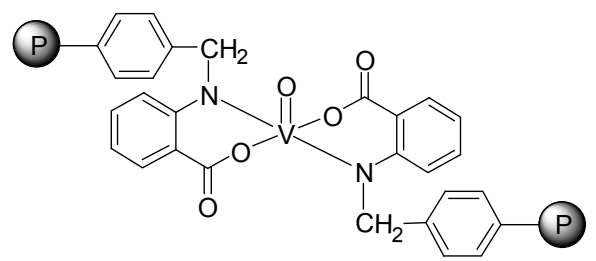

Scheme 8

2013 年, Zolfigol 等 ${ }^{[25]}$ 从简单易得的化合物出发, 通过简单的方法得到多相钒硅酸催化剂(SVA), $40 \mathrm{~min}$ 内可以将不同类型的硫醚氧化为亚砜(Eq. 6). 位阻大的 茮基苯基亚砜、二茮基亚砜、二苯基亚砜的产率均 $\geqslant$ 88\%. 二甲硫基甲烷氧化后，分离只得到了单硫氧化产 物. 该催化氧化体系除了催化剂可循环使用, 活性没有 明显降低外，反应也没有明显的放大效应，分别使用 5 , $10,50 \mathrm{mmol}$ 底物时, 产率只有很小幅度的降低.

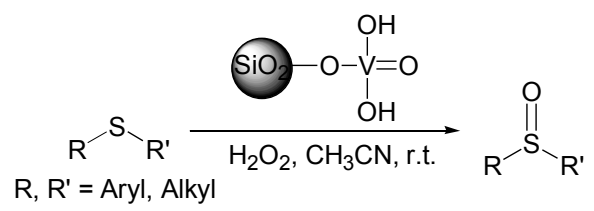

钒和一些手性配体形成的配合物，在合成手性亚砜 化合物中起着非常重要的作用 ${ }^{[26]}$. 2004 年 Ahn 等 ${ }^{[27]}$ 合成 了 4 种立体位阻大的手性席夫碱钒配合物(Scheme 9), 0 ${ }^{\circ} \mathrm{C} 、 \mathrm{CH}_{2} \mathrm{Cl}_{2}$ 溶剂中，在甲基芳基硫醚、茮基芳基硫醚的 氧化中，配合物 $4 \mathrm{a}$ 表现出优异的催化性能. 其中，茮基 苯基亚砜的立体选择性高达 99\%, 而且亚砜的主要构型 均为 $S$.

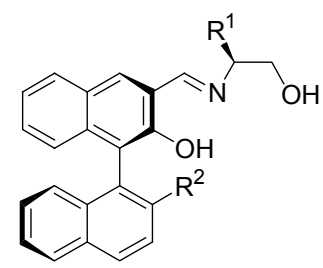

$(R, S)-4 \mathrm{a} \mathrm{R}^{1}=t-\mathrm{Bu}, \mathrm{R}^{2}=\mathrm{coO} t-\mathrm{Bu}$ $(R, S)-4 b R^{1}=t-B u, R^{2}=\mathrm{COOCH}_{3}$ $(R, S)-4 \mathrm{c} \mathrm{R} \mathrm{R}^{1}=t-\mathrm{Bu}, \mathrm{R}^{2}=\mathrm{OSiPh}_{2} t-\mathrm{Bu}$

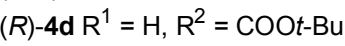


2004 年, Zhu 等 ${ }^{[28]}$ 发现可以利用同一种 salan-钒催 化体系、通过立体选择性氧化硫醚分别得到绝对构型为 $R$ 和 $S$ 构型的亚砜. $\mathrm{CHCl}_{3}$ 中, $0{ }^{\circ} \mathrm{C}$, 除间硝基苯基甲基亚 砜外, 其余亚砜均为 $R$ 构型, 而且 $e e$ 值为 $51 \% \sim 95 \%$. 奇怪的是, 温度升到 $25{ }^{\circ} \mathrm{C}$, 亚砜的构型主要为 $S$ 型, 虽 然 $e e$ 值为 $78 \% \sim 93 \%$, 但产率很低, 最高仅为 $32 \%$. 在 反应中, salan 配体和 $\mathrm{VO}(\mathrm{acac})_{2}$ 形成过氧钒配合物, 硫 原子进攻氧形成的中间体在过氧化氢的作用下, 给出产 物亚砜和过氧钒配合物, 从而完成催化循环(Scheme 10).

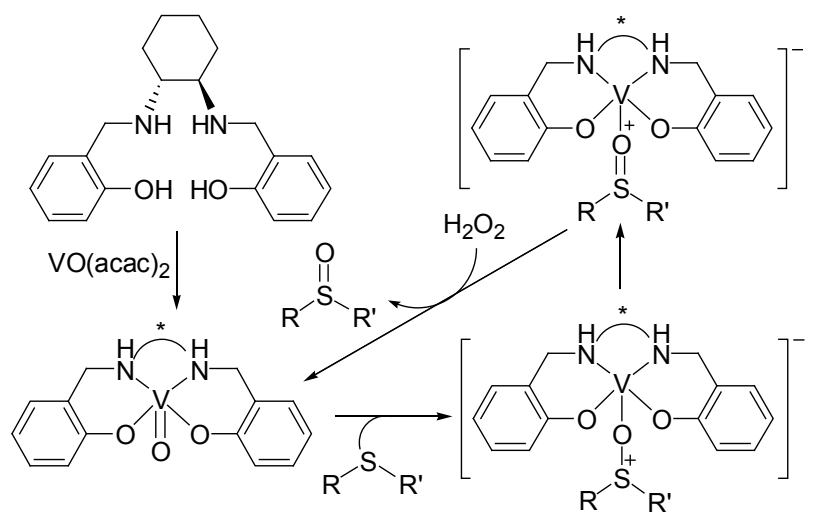

Scheme 10

2006 年, 高爱萍小组 ${ }^{[29}$ 报道了 $\mathrm{VO}(\mathrm{acac})_{2}$ 与二溴和 二碘取代的手性 Schiff 碱配体组成的催化体系(Scheme 11), 对甲基芳基硫醚的不对称氧化具有较高的催化活 性和中等至很高的对映选择性. 研究表明, 水杨醛 3,5 位、氨基醇手性碳上取代基的立体电子效应对反应的产 率和对映选择性有很大的影响. 其中配体 $\mathrm{L}^{3}$ 和 $\mathrm{L}^{4}$ 不仅 表现出较高的催化活性, 而且显示了高的对映选择性. 利用配体 $\mathrm{L}^{3}$ 和 $\mathrm{L}^{4}$, 甲基苯基亚砜的产率分别为 $79 \%$ 和 $84 \%, e e$ 值分别为 $81 \%$ 和 $88 \%$.

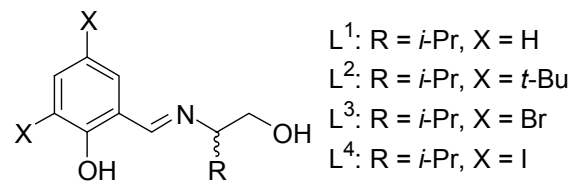

Scheme 11

2009 年, Liu 等 ${ }^{[30]}$ 通过 Suzuki-Miyaura 偶联合成了 一种手性的、带有刚性四氢喹啉的三齿配体化合物 (Scheme 12). 此配体在丙酮溶液中, 和 $\mathrm{VO}(\mathrm{acac})_{2}$ 结合 可以将甲基芳基硫醚以较高的产率(80\% 95\%)氧化为 亚砜, 产物的 $e e$ 值可以达到 77\%, 而且手性碳原子的构 型主要为 $S$ 型.

2011 年, Zeng 等 ${ }^{[31]}$ 合成了一系列取代基不同的手 性钒配合物(Scheme 13), 催化氧化烯丙基硫醚. 当 $\mathrm{R}^{1}$ 为 $\mathrm{CH}_{3}, \mathrm{R}^{2}$ 为 $\mathrm{I}$ 时, 苯基烯丙基亚砜的 $e e$ 值高达 $97 \%$, 但此<smiles>Cc1cc(-c2ccccc2O)nc2c1CCC[C@H]2O</smiles>

Scheme 12

体系的不足在于几种亚砜的产率均较低, 最高只有 $57 \%$.<smiles></smiles>

Scheme 13

2013 年, Romanowski 等 ${ }^{[32]}$ 从水杨醛和去甲麻黄素 出发, 通过简单的缩合得到三齿席夫碱配体 (Scheme 14), 进而和三乙氧基氧钒反应合成了一系列新颖的手 性氧桥双核钒配合物. 此催化剂在室温、 $\mathrm{CH}_{2} \mathrm{Cl}_{2}$-甲醇混 合溶剂中，可以催化过氧化氢选择性氧化硫醚 $(\mathrm{PhSR}$, $\mathrm{R}=\mathrm{Me}, \mathrm{Bz}$ )得到绝对构型主要为 $S$ 的亚砜. 虽然此体系 仅使用 $1 \mathrm{~mol} \%$ 的催化剂和 1.1 equiv.的过氧化氢, 就可 以得到产率为 $84 \% \sim 94 \%$ 的产物，但亚砜的 $e e$ 值不理 想，即使温度降至 $-20^{\circ} \mathrm{C}, e e$ 值最高仅为 $49 \%$.

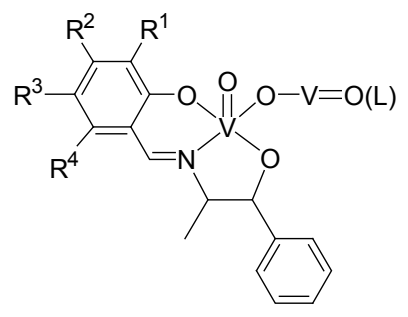

Scheme 14

\section{4 钛催化剂}

在一系列过氧化氢选择性氧化硫醚中, 钛催化剂如 TS-1 (MFI 型沸石), TS-2 (MEL 型沸石), Ti-b, Ti-MCM-41 (Ti-M41), Ti-HMS 等表现出了优良的催化 性能 ${ }^{[33]}$. 钛硅酸盐 TS-1 作为商品化的催化剂, 主要是 催化氧化小分子，而 Ti- $\beta$ 和钛孔材料可用于氧化大量硫

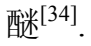

1996 年, Hulea 等 ${ }^{[35]}$ 比较了 $30{ }^{\circ} \mathrm{C}$ 下 Ti- $\beta$ zeolite 和 TS-1 催化氧化各种二烷基、二芳基硫醚的催化活性. 结 果表明: 在二乙基硫醚的氧化中, Ti- $\beta$ 和 TS- 1 的催化活 性相差不大. 而对于其它的硫醚, Ti- $\beta$ 的催化活性明显 强于 TS- 1 , 这可能归因于 Ti- $\beta$ 具有大的孔径.

2009 年, Bharadwaj 等 ${ }^{[36]}$ 利用磷酸浸渍二氧化钛的 方法制备了一种新型的由 $84.5 \%$ 的 $\mathrm{TiO}_{2}$ 和 $15.5 \%$ 的 
$\left[\mathrm{Ti}_{4} \mathrm{H}_{11}\left(\mathrm{PO}_{4}\right)_{9}\right] \bullet n \mathrm{H}_{2} \mathrm{O}(n=1 \sim 4)$ 组成的固体酸催化剂. 在 烷基芳基硫醚氧化为亚砜的反应中, 此催化剂表现出良 好的催化活性和选择性. 对氧化剂敏感的官能团如双 键、羟基、醛基等, 在氧化过程中不受影响. 但对于二 芳基、二烷基硫醚, 亚砜的选择性较低. 例如二丁基亚 砜、二甲基亚砜的选择性分别只有 72\%和 $20 \%$.

2009 年, Hulea 等 ${ }^{\left[{ }^{[3]}\right.}$ 成功合成了一种含钛 $1.5 \%$ 的有 序介孔钛硅材料 Ti-MCM-48. 并以乙腈为溶剂, $50{ }^{\circ} \mathrm{C}$ 下，催化剂催化氧化甲基苯基硫醚、二甲亚砜和二苯并 噻吩反应 $20 \mathrm{~min}$ 后, 甲基苯基硫醚、二甲亚砜的转化率 可以达到 $100 \%$. 但二苯并噻吩反应 $3 \mathrm{~h}$, 转化率仅有 $58 \%$.

2010 年, Rahimizadeh 等 ${ }^{[38]}$ 报道了纳米 $\mathrm{TiO}_{2}$ 和 Preyssler 杂多酸修饰的纳米 $\mathrm{TiO}_{2}$ 在硫醚氧化中的应用 (Scheme 15). 其中在乙腈中纳米 $\mathrm{TiO}_{2}$ 催化 2.5 equiv. $\mathrm{H}_{2} \mathrm{O}_{2}$ 可以将硫醚定量地转化为砜, 所用的 11 种底物中, 9 种产物的产率都达到 $100 \%$. 当采用 Preyssler 杂多酸修 饰的纳米 $\mathrm{TiO}_{2}$ 时, 催化剂的活性降低, 而选择性提高. 用此催化剂, 以异丙醇为溶剂, 依然用 2.5 equiv. $\mathrm{H}_{2} \mathrm{O}_{2}$, 硫醚可以被选择性地氧化为亚砜，产率为 $80 \% \sim 100 \%$.

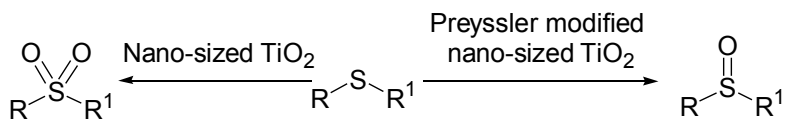

\section{Scheme 15}

利用非水解溶胶-凝胶法, Cojocariu 等 ${ }^{[39]}$ 制备了 $\mathrm{TiO}_{2}-\mathrm{SiO}_{2}$ 干凝胶. 此催化剂在二苯基硫醚、二苯并噻吩 和 4,6-二甲基二苯并噻吩的氧化反应中表现出非常好的 催化活性和选择性. $60{ }^{\circ} \mathrm{C}$ 、乙腈为溶剂, 位阻非常大的 4,6-二甲基二苯并噻吩可以 $80 \%$ 的转化率氧化为砜, 且 选择性达到 $99 \%$.

钛配合物也被应用于硫醚的氧化反应中. De Rosa 等 ${ }^{\left[{ }^{400}\right.}$ 合成了双菜基桥席夫碱钛配合物(Scheme 16), 无 溶剂条件下，仅使用 $0.5 \mathrm{~mol} \%$ 的催化剂和 1 equiv. 的过 氧化氢, 就可以将烷基芳基硫醚、二烷基硫醚在 $15 \mathrm{~min}$ 内选择性氧化为亚砜. 即使带有强吸电子基的 4-硝基苯 基甲基硫醚反应 $3 \mathrm{~h}$ ，产率也可以达到 74\%.

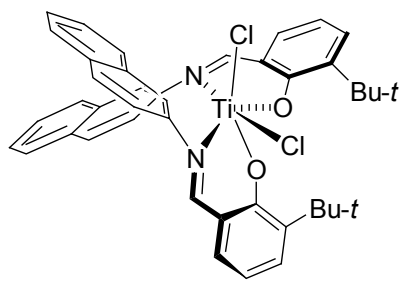

Scheme 16

2013 年, Talsi 等 ${ }^{[41]}$ 以 3,3'-二苯基取代的亚水杨基环
装物作为配体合成了一系列复杂的手性钛配合物, 用于 亚砜的不对称合成中, 并提出了可能的反应机理 (Scheme 17). 实验结果显示, 当芳环的 3,3'位连有大取 代基苯环所形成的催化剂, 在催化氧化位阻大的硫醚, 如芐基苯基硫醚时，表现出优异的立体选择性. 相反, 3,3'位带有小取代基的催化剂, 如 $\mathrm{CH}_{3}, \mathrm{OCH}_{3}$, 尤其是氢 原子时, 在位阻小的硫醚氧化中显示出了很高的立体选 择性. 利用 3,3'位带有弱电子受体的催化剂, 所有硫醚 转化为亚砜的反应不但有很高的 $e e$ 值 $(62 \% \sim 93 \%)$, 而 且选择性也高达 $92 \%$.

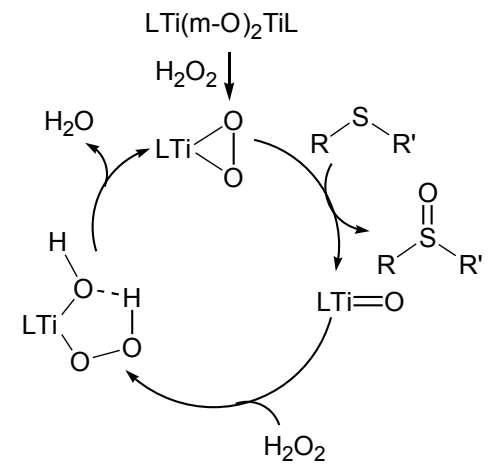

Scheme 17

\section{5 铁催化剂}

和其它金属相比，铁配合物在硫醚的氧化反应中的 研究报道较少. 但铁因具有无毒、价廉、易得等优点，近 年来逐渐引起化学工作者的关注 ${ }^{[42]}$. 商品化的四五氟 苯基卟啉铁(F20TPPFe)/过氧化氢是实验室中温和、快速 合成亚砜、砜的非常高效的催化氧化体系 ${ }^{[43]}$. 仅需 4 $\min 、 0.03 \% \sim 0.09 \%$ 的催化剂、 1 equiv. 的过氧化氢, 大 多数的亚砜可以得到 $90 \% \sim 95 \%$ 的分离收率. 苯环上带 有氧基吸电子基时，依然得到 93\%产率的亚砜．此外， 反应不受立体效应的影响，例如叔丁基苯基亚砜、二苯 基亚砜的收率都为 92\%; 底物中含有乙烯基、烯丙基、 羟基等对氧化剂敏感的基团，反应后均没有观察到氧化 产物. 调节催化剂和过氧化氢的用量分别到 $0.09 \%$ $0.25 \%, 2$ equiv., 反应 $15 \mathrm{~min}$, 硫醚可以被定量地氧化为 砜.

2007 年, Goldberg 等 ${ }^{[44]}$ 首次合成的氮杂取代卟啉铁 配合物也在二甲基、二苯基、芐基苯基、甲基苯基硫醚 选择性氧化为亚砜的反应中，显示了良好的活性，虽然 反应仅需要 $1 \mathrm{~min}$. 但需加配体 4- N,N -二甲基吡啶. 他们 认为在反应的过程中经历了 $\left[\mathrm{Fe}^{\mathrm{III}}-\mathrm{OOH}\right]$ 中间体.

2011 年, Rajabi 等 ${ }^{[45]}$ 将氧化铁纳米颗粒负载于 SBA-15 分子篮上, 发现催化剂在纯水介质中, 可以将 硫醚选择性氧化为亚砜，产物的产率大于 $95 \%$. 尤为重 要的是催化剂具有长的使用寿命, 重复利用 10 次, 催化 
性能没有明显降低. 但和其它反应体系不同的是, 此体 系使用过量的硫醚，而非价廉的过氧化氢.

采用简单的 $\mathrm{FeCl}_{3}$ 和 $\mathrm{FeBr}_{3}$ 作催化剂, 室温、乙腈中, Ren 等 ${ }^{[46]}$ 将二苯基硫醚和甲基苯基硫醚选择性的氧化 为亚砜. 其中, $\mathrm{FeBr}_{3}$ 的催化活性强于 $\mathrm{FeCl}_{3}$.

铁和一些手性配体形成的配合物, 在硫醚的手性合 成中, 也表现出很好的催化活性 ${ }^{[4]}$. Bolm 等 ${ }^{[48]}$ 报道了原 位形成的 $\mathrm{Fe}$-席夫碱配合物, 可以催化过氧化氢氧化硫 醚给出 $S$ 构型的亚砜(Eq. 7), 但是亚砜的产率 $\leqslant 44 \%, e e$ 值也只有中等水平. 随后, 他们尝试在反应体系中加入 取代苯甲酸及其盐类添加剂, 以期提高产率和对映选择 性. 和取代苯甲酸盐相比 ${ }^{[49]}$, 体系中加入取代苯甲酸添 加剂 ${ }^{[50]}$, 亚砜的产率和 $e e$ 值提高的幅度比较大, 分别可 以达到 36\% 78\%和 66\% 95\%.

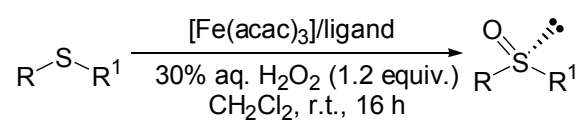

2007 年 Katsuki 等 ${ }^{[51]}$ 发现 Fe-salan 配合物在烷基芳 基、二烷基硫醚的不对称氧化反应中显示出非常优异的 催化性能(Scheme 18). 当使用 1.5 equiv. 的过氧化氢, 纯 水介质中、 $20{ }^{\circ} \mathrm{C}$ 下仅反应 $3 \mathrm{~h}$, 就可以得到产率为 $76 \% \sim 91 \%, e e$ 值为 $81 \% \sim 96 \%$ 的亚砜.

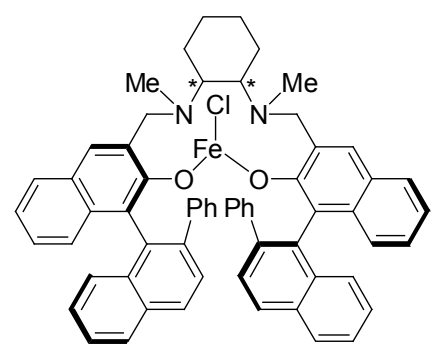

Scheme 18

水溶性的铁-卟啉配合物在水一甲醇混合溶剂中 (Scheme 19), 催化过氧化氢氧化硫醚取得了良好的效 果 ${ }^{[52]}$. 除甲基芐基硫醚外, 甲基芳基硫醚的转化率 $\geqslant$ $98 \%$, 亚砜的化学选择性 $\geqslant 93 \%, e e \geqslant 68 \%$.

和均相催化剂相比, 固载手性催化剂的催化活性和 立体选择性均较低, 因此, 有关负载手性催化剂在硫醚 不对称合成反应中鲜有报道. 2011 年, $\mathrm{Li}$ 等 ${ }^{[53]}$ 将手性 Fe-Salan 配合物载于介孔二氧化硅纳米笼中, 并取得了 很好的效果, 硫醚的转化率和亚砜的选择性均高于 $84 \%$, 产物的 $e e$ 值也可以达到 $73 \% \sim 85 \%$. 可能归因于 过氧化氢在两亲性纳米笼催化剂中能快速扩散, 所以其 立体选择性明显高于脂溶性、水溶性的纳米笼催化剂. 固载催化剂重复使用 4 次, 催化效果没有明显降低.

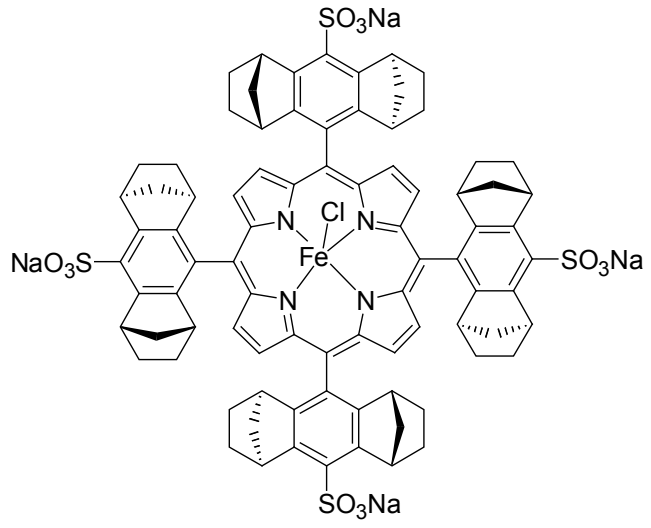

Scheme 19

\section{6 其它金属催化剂}

除了上述的金属外，其它一些金属也可催化过氧化 氢氧化硫醚. 常见的有锌、锰、铜、碲等 ${ }^{[54]} .2009$ 年, Sharma 等 ${ }^{[55]}$ 发现 $\mathrm{ZnO} /$ 聚苯胺复合材料在无溶剂条件下, 可以催化过氧化氢选择性地氧化硫醚为亚砜, 且仅使用 1 equiv.的过氧化氢(Eq. 8). 催化剂重复利用 4 次, 催化 活性没有明显的降低. 但此体系对于长链的二烷基硫醚 以及芳环上连有吸电子基的烷芳基硫醚的催化效果不 好.

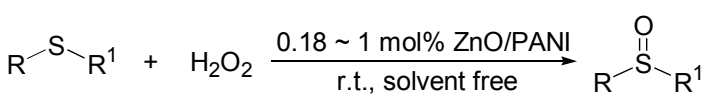

$$
\begin{aligned}
& \mathrm{R}=\text { Aryl, Alkyl; } \mathrm{R}^{1}=\text { Alkyl }
\end{aligned}
$$

以四氢呋喃作溶剂, 三氟甲磺酸铈可以催化 $50 \%$ 的 过氧化氢选择性地氧化硫醚为亚砜风 ${ }^{[56]}$. 含有醇、醛、烯 烃、卤素、腈和酯等官能团的底物, 在反应过程中均不 受影响, 而且亚砜产率为 $73 \% \sim 96 \%$. 催化剂在反应过 程中可能经历了如下两种结构的中间体(Scheme 20).
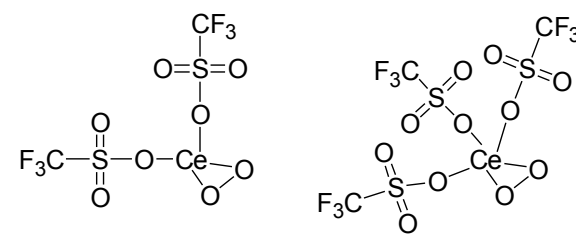

Scheme 20

与其他金属相比, $\mathrm{Mn}(\mathrm{III})$ 因容易和杂原子配位形成 功能特殊的配合物, 而在催化氧化硫醚反应中的应用较 多 ${ }^{[57]} .2006$ 年, Hosseinpoor 等 ${ }^{[58]}$ 用锰席夫碱配合物在乙 酸溶液中催化过氧化氢选择性氧化硫醚为亚砜(Scheme 21). 实验结果表明，除二芐基硫醚外，其余硫醚在 35 min 内的转化率均可达到 $100 \%$, 亚砜的产率也高达 $84 \% \sim 100 \%$. 但此氧化反应需要冰醋酸作溶剂, 还要消 耗 4 equiv.的氧化剂. 


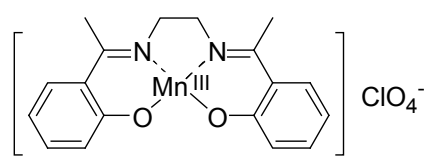

Scheme 21

2012 年, Abdolmaleki 等 ${ }^{[59]}$ 以部分水溶性的 Mn(III)salen 为催化剂(Scheme 22), 在水-异辛烷两相体系中催 化过氧化氢, 选择性地氧化硫醚为亚砜. 虽然底物的转 化率均达到 $100 \%$, 亚砜的产率在 $87 \%$ 100\%间, 但反 应需 8 equiv.的过氧化氢.

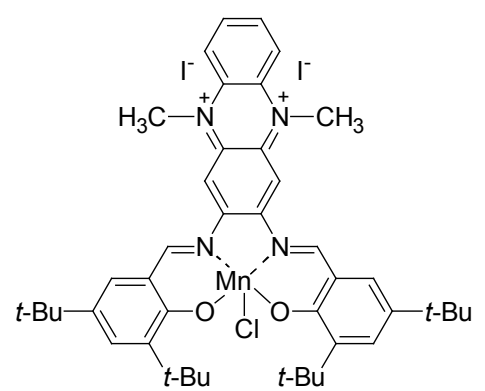

Scheme 22

2013 年, Sroura 等 ${ }^{[60]}$ 合成了可溶于水的手性卟啉锰 催化剂(Scheme 23), $25{ }^{\circ} \mathrm{C}$, 甲醇、水混合溶剂中, 催化 过氧化氢氧化前手性甲基苯基硫醚及其衍生物. 亚砜的 产率在 $43 \% \sim 83 \%$, 但 $e e$ 值仅有 5\% 57\%. 该催化氧化 体系成功地应用于消炎、抗癌药物苏灵大(亚磺酰茚酷 酸)的制备中.

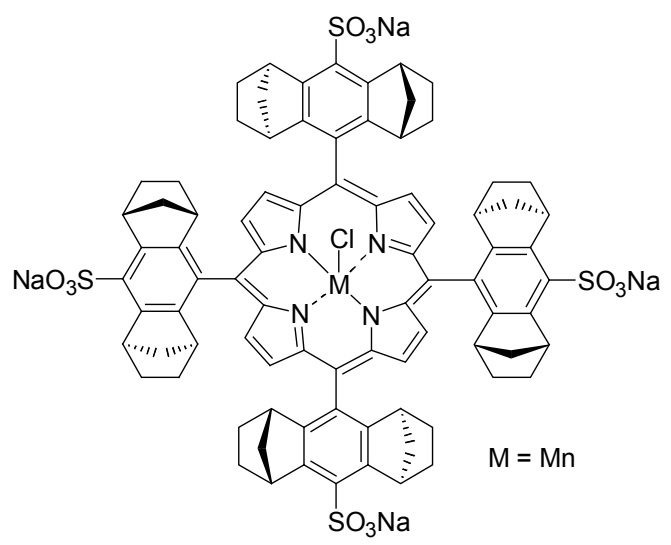

Scheme 23

最近, Islam 等 ${ }^{[61]}$ 通过配位的方式将二价铜固载到 聚苯乙烯载体上(Scheme 24), 并发现该催化剂在室温 下、乙腈溶剂中, 可以催化过氧化氢选择性的氧化硫醚 为亚砜. 此催化剂重复使用六次后, 活性没有明显降低, 但硫醚的转化率较低, 最高只有 $91 \%$.

\section{7 非金属催化剂}

除了综上所述的金属催化剂催化过氧化氢氧化硫<smiles></smiles>

Scheme 24

醚的反应之外, 少量非金属催化剂也被应用于过氧化氢 的氧化反应中. 2010 年, Bahrami 等 ${ }^{[62]}$ 首次报道了三甲基 氯硅烷作为硫醚氧化反应的催化剂. 室温下, 乙腈为溶 剂, 2 35 min 内, 该催化剂可以将一系列二烷基、二芳 基、烷基芳基硫醚高产率的氧化为相应的亚砜，亚砜产 率均大于 $90 \%$. 同时, 他们认为在反应中，过氧化氢首 先进攻三甲基氯硅烷形成过氧活性中间体，继而硫醚进 攻亲电性增强的氧原子并释放氢质子给出亚砜产物 (Scheme 25).

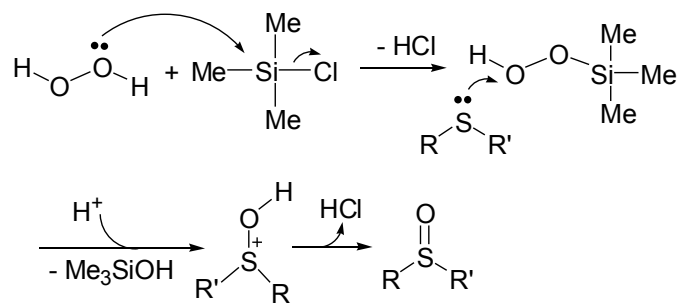

Scheme 25

2010 年, Shokrolahi 等 ${ }^{[63]}$ 利用磺化后的多孔介质炭 作为硫醚氧化反应的多相催化剂. 室温、无溶剂条件下, 通过改变过氧化氢的用量, 可以将氧化反应停留在亚砜 或者砜. 利用 1 equiv.的过氧化氢，除吩噁嗪，其余的硫 醚都可以在 $1 \mathrm{~h}$ 内被高效的氧化为亚砜(产率 $\geqslant 85 \%$ ). 当 过氧化氢用量增加到 4 equiv. 时, 反应 $1 \mathrm{~h}$ 内, 砜的产率 均 $\geqslant 87 \%$. 在反应中, 过氧化氢与磺酸形成了过氧磺酸, 所以有效地提高了过氧化氢的氧化能力 (Scheme 26).

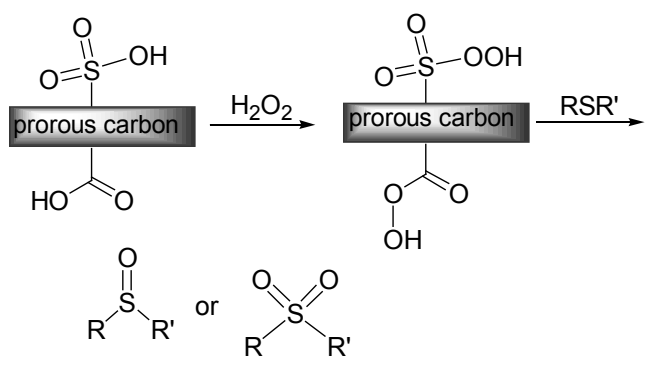

Scheme 26

2012 年, Tumula 等 ${ }^{[64]}$ 发现乙酸和大孔树脂-15 催化 过氧化氢氧化硫醚是制备砜的一种简单高效的方法. 在 $50{ }^{\circ} \mathrm{C}$ 时，二烷基、二芐基、二苯基、烷基芳基、苠基芳 基、烷基茮基硫醚的转化率均 $>99 \%$ ，相应砜的选择性 也都 $>99 \%$. 而且根据反应所需时间推断出硫醚的反应 
活性和底物的亲核性有关: 二烷基硫醚 $>$ 二芐基硫醚> 二苯基硫醚. 大孔树脂- 15 重复利用 6 次之后，甲基苯基 砜的产率仅降低 $6.8 \%$. 但是此催化体系需要大量的乙 酸, $1 \mathrm{mmol}$ 底物, 需要 $5.7 \mathrm{~mL}$ 的乙酸.

小分子有机催化剂因具有易操作、高效的特点, 在 氧化反应中也不断的发展, 而且是有机催化研究的一个 热点. Lindén 等 ${ }^{[65]}$ 用黄素在甲醇溶液中选择性地氧化烯 丙基硫醚和乙烯基硫醚为相应的亚砜. 催化体系对烯丙 基硫醚的氧化有很好的效果, $2.5 \sim 3 \mathrm{~h}$ 内, 利用 1.5 equiv. 的过氧化氢, 各种硫醚均得到满意的氧化产率. 底物中的双键、参键、羟基不受影响. 但催化体系对于 乙烯基硫醚的氧化反应不是很理想, 当过氧化氢的用量 增加至 10 倍时, 乙烯基亚砜的产率最高也仅是 $60 \%$. 反 应时, 催化剂前体 $N, N, N-1,3,5$-三烷基黄素在氧气的活 化下形成过氧中间体, 亲电性的氧原子从中间体转移到 硫醚给出产物, 去氧中间体再被过氧化氢氧化从而维持 催化循环(Scheme 27).

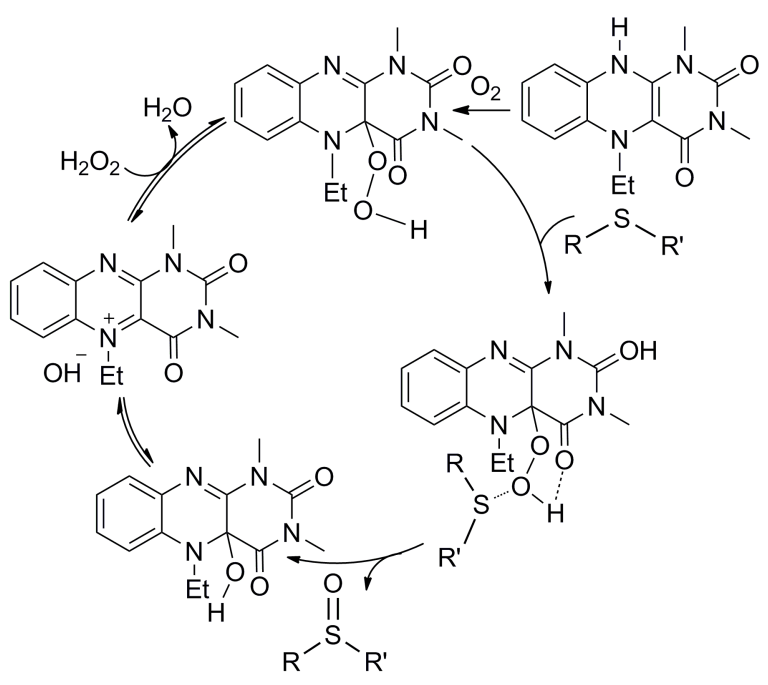

\section{Scheme 27}

2006 年, Lindén 小组 ${ }^{[6]}$ 又在甲醇溶液中, 将缺电子 的黄素和离子液体结合起来催化过氧化氢氧化硫醚 $(\mathrm{Eq}$. 9). 在室温下, 该催化剂可以催化过氧化氢将富电子或 缺电子的硫醚在 1.5 6h 内氧化到相应的亚砜, 且产率 较高. 而且此催化剂循环使用 7 次后, 反应的选择性和 产率没有明显的降低.

2010 年, Marsh 等 ${ }^{[67]}$ 提出了一种桥联的四环黄素有 机催化剂(Scheme 28). 室温下, 甲醇为溶剂, 将硫醚选 择性地氧化为亚砜, 反应后没有过度氧化产物砜被检测 出. 此有机催化剂在反应中表现出良好的催化活性, 10 种硫醚的氧化产率均大于 $89 \%$.

2010 年, Bakavoli 等 ${ }^{[68}$ 采用 $\mathrm{DBUH}-\mathrm{Br}_{3}$ 为催化剂, 室温下、乙腈溶剂中, 短时间内催化过氧化氢将 13 种硫
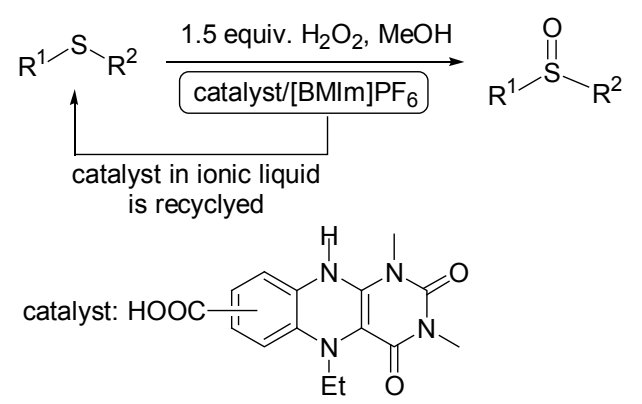<smiles></smiles>

Scheme 28

醚选择性地氧化为亚砜. 此催化氧化体系不但显示出良 好的催化活性，而且具有高的选择性. 除 2-羟乙基苯基 亚砜产率较低，为 $66 \%$ 外，其它亚砜的产率为 $75 \%$ 95\% . 底物中含有易于被氧化的基团，如羟基、羰基、 双键等官能团, 在反应中均不受影响. 同时, 他们提出 了反应的可能机理(Scheme 29).

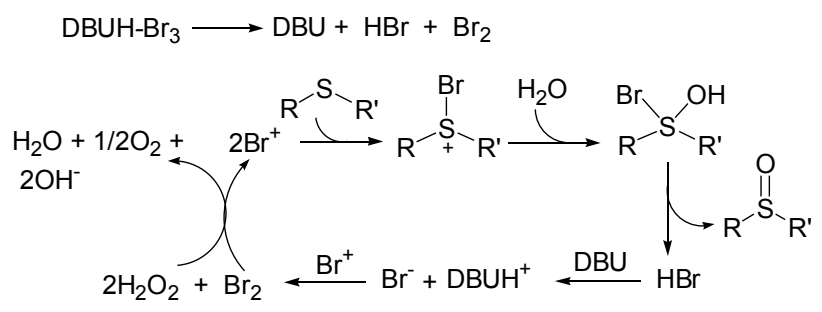

Scheme 29

2010 年, 室温、无溶剂条件下, Rostami 等 ${ }^{[69]}$ 通过改 变过氧化氢的用量, 用硼酸催化过氧化氢, 短时间内可 以将硫醚选择性、高产率地氧化为亚砜 $(85 \%$ \% $95 \%)$ 或 砜( $87 \% \sim 95 \%$ ) (Eq. 10). 底物中的羟基、酯基和醛基等 活性基团在反应中不受影响. 值得一提的是，与其它催 化氧化体系不同, 利用此体系, 位阻大的二苯基硫醚的 反应活性高于其它硫醚. 有趣的是，3-甲硫基丙醛和 3甲硫基丙酸乙酯迅速反应得到亚砜，但当过氧化氢量增 加时, 却检测不到砜.

$$
\begin{aligned}
& \mathrm{R}^{1}-\mathrm{S}_{-} \mathrm{R}^{2} \stackrel{\text { boric acid (cat.), } \mathrm{H}_{2} \mathrm{O}_{2}}{\text { solvent-free, r.t. }} \mathrm{R}^{1}-\mathrm{S}_{-\mathrm{R}^{2}}+\mathrm{R}^{1}-\mathrm{S}_{\mathrm{R}^{2}}^{\prime \prime}(10) \\
& \mathrm{R}^{1}, \mathrm{R}^{2}=\mathrm{Ar} \text {, benzylic, linear, cyclic }
\end{aligned}
$$

Kraus 等 ${ }^{\left[{ }^{700}\right.}$ 合成了一系列黄素、异咯嗪-环糊精共价 复合物作为亚砜立体选择性合成的催化剂, 并逐一对催 化剂的活性进行了研究. 结果发现在富电子甲基苯基硫 醚的氧化反应中, $\beta$-环糊精一黄素复合物的催化效果突 
出(Scheme 30), 水介质中, 用磷酸盐调节 $\mathrm{pH}$ 值, 产物的 $e e$ 值可以达到 $80 \%$.

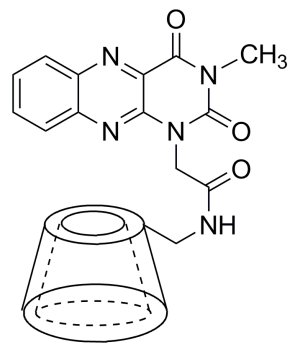

Scheme 30

2011 年, Huang 等 ${ }^{[71]}$ 合成了含氟硫嫝催化剂. 二氯 甲烷为溶剂, 实现了此有机催化剂催化氧化硫醚为亚砜 的反应(Eq. 11). 几种底物得到较理想的产率, 例如二苯 基硫醚经柱色谱分离后得到 76\%的亚砜. 催化剂经过简 单分离后可再循环利用, 循环第五次时, 甲基苯基亚砜 产率依然可达 $89 \%$. 但此催化氧化体系需要较长的反应 时间, 二烷基硫醚需反应 $8 \mathrm{~h}$, 其它的硫醚反应均超过 $10 \mathrm{~h}$.

$$
\text { catalyst: }
$$

近年来，一些手性有机催化剂也逐渐被发现并用于 亚砜的不对称合成中. 2012 年, Tao 等 ${ }^{[72]}$ 从 $R-1,1^{1}$-双荟 基-2,2'-二醇出发, 合成了手性磷酸催化剂(Scheme 31), 可以将烃基芳基硫醚、环状二硫缩醛选择性地氧化为中 等及其以上产率、ee 值的亚砜. 虽然他们第一次将很强 的质子酸应用于亚砜的不对称合成中, 反应中也观察不 到过度氧化产物, 但反应需 $50 \%$ 的过氧化氢, 在 $-40{ }^{\circ} \mathrm{C}$ 下反应 $48 \sim 144 \mathrm{~h}$. 同年, List 等 ${ }^{[73]}$ 发展了一种类似结构 的手性磷酸催化剂, 反应 $2 \sim 8 \mathrm{~h}$, 亚砜的产率和 $e e$ 值分 别可以达到 $\geqslant 89 \%$ 和 $\geqslant 85 \%$, 他们将此体系成功用于非 甾体抗炎镇痛药舒林酸的合成中.

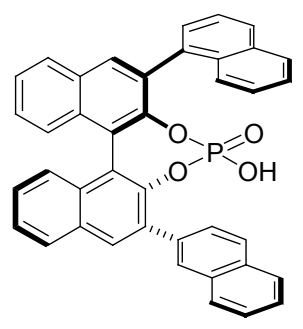

Scheme 31
2012 年, Yashima 等 ${ }^{[74]}$ 从核黄素出发, 通过包含自 组装等多步方法合成了一种具有超分子扭曲状、螺旋结 构的手性高分子化合物, 此催化剂虽然可以将含硫底物 选择性氧化为亚砜，但产率仅达到中等水平, ee 值最高 仅有 $60 \%$.

\section{7 总结和展望}

综上所述, 在硫醚选择性氧化过程中, 过氧化氢以 其低毒, 价廉, 绿色环保等优点作为硫醚氧化的氧源而 深受科研工作者的关注. 近年发展了许多高效催化过氧 化氢的均相、多相催化剂. 但是大多数反应都在有机溶 剂中进行，仅有几例关于无溶剂或者绿色介质中进行硫 醚氧化反应的报道. 随着原子经济性反应、绿色化学以 及可持续化学概念的提出, 使用清洁的氧化剂, 选择高 效的催化剂和有效的反应途径，在温和的条件下进行反 应, 将是实现硫醚氧化反应绿色化的重要研究课题.

\section{References}

[1] (a) Maurya, M. R.; Chandrakar, A. K.; Chand, S. J. Mol. Catal. A: Chem. 2007, 274, 192.

(b) Nicolaou, K. C.; Magolda, R. L.; Sipio, W. J.; Barnette, W. E.; Lysenko. Z.; Joullie. M. M. J. Am. Chem. Soc. 1980, 102, 3784.

(c) Kropp, P. J.; Breton, G. W.; Fields, J. D.; Tung, J. C.; Loomis, B. R. J. Am. Chem. Soc. 2000, 122, 4280.

(d) Breton, G. W.; Fields, J. D.; Kropp, P. J. Tetrahedron Lett. 1995, 36,3525 .

(e) Bonadies, F.; De Angelis, F.; Locati, L.; Scettri, A. Tetrahedron Lett. 1996, 37, 7129.

(f) Venier, C. G.; Squires, T. G.; Chen, Y. Y.; Hussmann, G. P.; Shei, J. C.; Smith, B. F. J. Org. Chem. 1982, 47, 3774.

[2] (a) Bortolini, O.; Campestrini, S.; Di Furia, F.; Modena, G. J. Org. Chem. 1987, 52, 5093.

(b) Ballistreri, F. P.; Bazzo, A.; Tomaselli, G. A.; Toscano, R. M. J. Org. Chem. 1992, 57, 7074.

[3] (a) Neumann, R.; Juwiler, D. Tetrahedron 1996, 52, 8781.

(b) Gresley, N. M.; Griffith, W. P.; Laemmel, A. C.; Nogueira, H. I. S.; Parkin, B. C. J. Mol. Catal. A: Chem. 1997, 117, 185.

(c) Collins, F. M.; Lucy, A. R.; Sharp, C. J. Mol. Catal. A: Chem. 1997, 117, 397.

[4] Sato, K.; Hyodo, M.; Aoki, M.; Zheng, X.-Q.; Noyori, R. Tetrahedron 2001, 57, 2469.

[5] Gharah, N.; Chakraborty, S.; Mukherjee, A.; Bhattacharyya, R. Inorg. Chim. Acta 2009, 362, 1089.

[6] Xue, X. L.; Zhao, W.; Ma, B. C.; Ding, Y. Catal. Commun. 2012, $26,73$.

[7] Das, S. P.; Boruah, J. J.; Chetry, H.; Islam, N. S. Tetrahedron Lett. 2012, 53, 1163.

[8] Koo, D. H.; Kim, M.; Chang, S. Org. Lett. 2005, 7, 5015.

[9] Karimi, B.; Ghoreishi-Nezhad, M.; Clark, J. H. Org. Lett. 2005, 7, 625.

[10] Shi, X. Y.; Ma, W. J.; Han, X. Y.; Zhang, W.; Lu, A. F.; Wei, J. F. Chem. J. Chin. Univ. 2011, 32, 2321 (in Chinese).

(石先芗，马文娟，韩晓燕，张文，吕爱风，魏俊发，高等学校化 学学报, 2011, 32, 2321.)

[11] Shi, X. Y.; Ma, W. J.; Ou, H.; Han, X. Y.; Lu, C. M.; Chen, Y.; Wei, 
J. F. J. Braz. Chem. Soc. 2012, 23, 1536.

[12] Jeyakumar, K.; Chakravarthy, R. D.; Chand, D. K. Catal. Commun. 2009, 10, 1948.

[13] Tundo, P.; Romanelli, G. P.; Vazquez, P. G.; Arico, F. Catal. Commun. 2010, 11, 1181.

[14] Romanelli, G. P.; Villabrille, P. I.; Caceres, C. V.; Vazquez, P. G.; Tundo, P. Catal. Commun. 2011, 12, 726.

[15] Raghavan, P. S.; Ramaswamy, V.; Upadhya, T. T.; Sudalai, A.; Ramaswamy, A. V.; Sivasanker, S. J. Mol. Catal. A: Chem. 1997, $122,75$.

[16] Sharma, R. K.; Pandey, A.; Gulati, S. Polyhedron 2012, 45, 86.

[17] Grivani, G.; Gholampoor, N. J. Iran. Chem. Soc. 2012, 9, 349.

[18] Anisimov, A. V.; Fedorova, E. V.; Lesnugin, A. Z.; Senyavin, V. M.; Aslanov, L. A.; Rybakov, V. B.; Tarakanova, A. V. Catal. Today 2003, 78, 319 .

[19] Smith, T. S.; Pecoraro, V. L. Inorg. Chem. 2002, 41, 6754.

[20] Berrocal, T.; Larrea, E. S.; Iglesias, M.; Arriortua, M. R. J. Mol. Catal. A: Chem. 2011, 335, 176.

[21] Hussain, S.; Talukdar, D. S.; Bharadwaj, K.; Chaudhur, M. K. Tetrahedron Lett. 2012, 53, 6512.

[22] Al-Hashimi, M.; Fisset, E.; Sullivan, A. C.; Wilson, J. R. H. Tetrahedron Lett. 2006, 47, 8017.

[23] Prasanth, K. L.; Maheswaran, H. J. Mol. Catal. A: Chem. 2007, $268,45$.

[24] Islam, S. M.; Roy, A. S.; Mondal, P.; Salam, N. J. Mol. Catal. A: Chem. 2012, 358, 38.

[25] Zolfigol, M. A.; Khazaei, A.; Safaiee, M.; Mokhles, M.; Rostamiana, R.; Bagheri, M.; Shiri, M.; Krugere, H. G. J. Mol. Catal. A: Chem. 2013, 370, 80.

[26] (a) Tang, T. P.; Volkman, S. K.; Ellman, J. A. J. Org. Chem. 2001, 66, 8772 .

(b) Yuste, F.; Ortiz, B.; Carrasco, A.; Peralta, M.; Quintero, L.; Sanchez-Obregon, R.; Walls, F.; Garcia Ruano, J. L. Tetrahedron: Asymmetry 2000, 11, 3079.

(c) Vetter, A. H.; Berkessel, A. Tetrahedron Lett. 1998, 39, 1741.

(d) Ando, R.; Yagyu, T.; Maeda, M. Inorg. Chim. Acta 2004, 357, 2237.

[27] Jeong, Y.-C.; Choi, S.; Hwang, Y. D.; Ahn, K.-H. Tetrahedron Lett. 2004, 45, 9249.

[28] Sun, J.; Zhu, C.; Dai, Z.; Yang, M.; Pan, Y.; Hu, H. J. Org. Chem. 2004, 69, 8500 .

[29] Gao, A. P.; Wang, M.; Wang, D. P.; Zhang, L.; Liu, H. B.; Tian, W.; Sun, L. C. Chin. J. Catal. 2006, 27, 743 (in Chinese). (高爱萍, 王梅, 王东平, 张路, 刘海涁, 田伟, 孙立成, 催化学 报, 2006, 27, 743.)

[30] Liu, H. B.; Wang, M.; Wang, Y.; Wang, Y.; Sun, H.; Sun, L. C. Catal. Commun. 2009, 11, 294.

[31] Zeng, Q. L.; Gao, Y. X.; Dong, J. Y.; Weng, W.; Zhao, Y. F. Tetrahedron: Asymmetry 2011, 22, 717.

[32] (a) Romanowski, G.; Wera, M. Polyhedron 2013, 50, 179. (b)Romanowski, G. J. Mol. Catal. A: Chem. 2013, 368 369, 137.

[33] (a) Raghavan, P. S.; Ramaswamy, V.; Upadhya, T. T.; Sudalai, A.; Ramaswamy, A. V.; Sivasanker, S. J. Mol. Catal. A: Chem. 1997, 122,75 .

(b) Raju, S. V. N.; Upadhya, T. T.; Ponatnam, S.; Daniel, T.; Sudalai, A. Chem. Commun. 1996, 16, 1969.

(c) Moreau, P.; Hulea, V.; Gomez, S.; Brunel, D.; Renzo, F. D. Appl. Catal. A: Gen. 1997, 155, 253.

(d) Corma, A.; Iglesias, M.; Sanchez, F. Catal. Lett. 1996, 39, 153. (e) Kholdeeva, O. A.; Derevyankin, A. Y.; Shmakov, A. N.; Trukhan, N. N.; Paukshtis, E. A.; Tuel, A.; Romannikov, V. N. $J$. Mol. Catal. A: Chem. 2000, 158, 417. (f) Reddy, R. S.; Reddy, J. S.; Kumar, R.; Kumar, P. J. Chem. Soc. Chem. Commun. 1992, 2, 84.

[34] Pitchen, P.; Dunach, E.; Deshmukh, M. N.; Kagan, H. B. J. Am. Chem. Soc. 1984, 106, 81.

[35] Hulea, V.; Oreau, P.; Di Renzo, F. J. Mol. Catal. 1996, 111, 325.

[36] Bharadwaj, S. K.; Sharma, S. N.; Hussain, S.; Chaudhuri, M. K. Tetrahedron Lett. 2009, 50, 3767.

[37] Hulea, V.; Maciuca, A-L.; Cojocariu, A-M.; Ciocan, C-E.; Dumitriu, E. C. R. Chimie 2009, 12, 723.

[38] Rahimizadeh, M.; Rajabzadeh, G.; Khatami, S-M.; Eshghi, H.; Shiri, A. J. Mol. Catal. A: Chem. 2010, 323, 59.

[39] (a) Cojocariu, A. M.; Mutin, P. H.; Dumitriu, E.; Fajula, F.; Vioux, A.; Hulea, V. Appl. Catal. B: Environ. 2010, 97, 407.

(b) Cojocariu, A. M.; Mutin, P. H.; Dumitriu, E.; Aboulaich, A.; Vioux, A.; Fajula, F.; Hulea, V. Catal. Today 2010, 157, 270.

[40] De Rosa, M.; Lamberti, M.; Pellecchia, C.; Scettri, A.; Villano, R.; Soriente, A. Tetrahedron Lett. 2006, 47, 7233.

[41] Talsi, E. P.; Bryliakov, K. P. Appl. Organomet. Chem. 2013, 27, 239.

[42] (a) Duboc-Toia, C.; Ménage, S.; Ho, R. Y. N.; Que, L.; Lambeaux, C.; Fontecave, M. Inorg. Chem. 1999, 38, 1261.

(b) Kaczorowska, K.; Kolarska, Z.; Mitka, K.; Kowalski, P. Tetrahedron 2005, 61, 8315 .

(c) Jayaseeli, A. M. I.; Rajagopal, S. J. Mol. Catal. A: Chem. 2009, 309, 103.

[43] Baciocchi, E.; Gerini, M. F.; Lapi, A. J. Org. Chem. 2004, 69, 3586.

[44] Kerber, W. D.; Ramdhanie, B.; Goldberg, D. P. Angew. Chem., Int. Ed. 2007, 46, 3718.

[45] Rajabi, F.; Naserian, S.; Primo, A.; Luque, R. Adv. Synth. Catal. 2011, 353, 2060.

[46] Villalobos, L.; Ren, T. Inorg. Chem. Commun. 2013, 28, 52.

[47] Mekmouche, Y.; Hummel, H.; Ho, R. Y. N.; Que, L.; Schünemann, V.; Thomas, F. Chem. Eur. J. 2002, 8, 1196.

[48] Legros, J.; Bolm, C. Angew. Chem., Int. Ed. 2003, 42, 5487.

[49] Legros, J.; Bolm, C. Chem. Eur. J. 2005, 11, 1086.

[50] Legros, J.; Bolm, C. Angew. Chem., Int. Ed. 2004, 43, 4225.

[51] Egami, H.; Katsuki, T. J. Am. Chem. Soc. 2007, 129, 8940.

[52] Maux, P. L.; Simonneaux, G. Chem. Commun. 2011, 47, 6957.

[53] Li, B.; Bai, S.; Wang, P.; Yang, H.; Yang, Q.; Li, C. Phys. Chem. Chem. Phys. 2011, 13, 2504.

[54] (a) Velusamy, S.; Kumar, A. V.; Saini, R.; Punniyamurthy, T. Tetrahedron Lett. 2005, 46, 3819.

(b) Kadnikova, E. N.; Kostic, N. M. J. Org. Chem. 2003, 68, 2600.

(c) Liu, R.; Wu, L. Z.; Feng, X. M.; Zhang, Z.; Li, Y. Z.; Wang, Z. L. Inorg. Chim. Acta 2007, 360, 656.

(d) Kim, K. S.; Hwang, H. J.; Cheong, C. S.; Hahn, C. S. Tetrahedron Lett. 1990, 31, 2893.

(e) Chambers, R. D.; Clark, M. Tetrahedron Lett. 1970, 32, 2741.

(f) Kirihara, M.; Yamamoto, J.; Noguchi, T.; Itou, A.; Naito, S.; Hirai, Y. Tetrahedron 2009, 65, 10477.

[55] Sharma, S. P.; Suryanarayana, M. V. S.; Nigam, A. K.; Chauhan, A. S.; Tomar, L. N. S. Catal. Commun. 2009, 10, 905

[56] Rama Raju, B.; Sarkar, S.; Chandramoulali Reddy, U.; Saikia, A. K. J. Mol. Catal. A: Chem. 2009, 308, 169.

[57] (a) Brinksma, J.; La Crois, R.; Feringa, B. L.; Donnoli, M. I.; Rosini. C. Tetrahedron Lett. 2001, 42, 4049.

(b) Chen, M.; Patkar, L. N.; Lin, C. J. Org. Chem. 2004, 69, 2884.

(c) Barker, J. E.; Ren, T. Tetrahedron Lett. 2004, 45, 4681.

(d) Mirkhani, V.; Tangestaninejad, S.; Moghadam, M.; Mohammadpoor-Baltork, I.; Kargar, H. J. Mol. Catal. A: Chem. 2005, 242, 251. 
[58] Hosseinpoor, F.; Golchoubian, H. Tetrahedron Lett. 2006, 47, 5195.

[59] Abdolmaleki, A.; Malek-Ahmadi, S. J. Iran. Chem. Soc. 2012, 9, 1015.

[60] Sroura, H.; Jalkha, J.; Mauxa, P. L.; Chevancea, S.; Kobeissib, M.; Simonneauxa, G. J. Mol. Catal. A: Chem. 2013, 370, 75.

[61] Islam, S. K. M.; Roy, A. S.; Mondal, P.; Salam, N.; Paul, S. Catal. Lett. 2013, 143, 225.

[62] Bahrami, K.; Khodaei, M. M.; Yousefi, B. H.; Arabi, M. S. Tetrahedron Lett. 2010, 51, 6939.

[63] Shokrolahi, A.; Zali, A.; Keshavarz, M. H. Chin. J. Catal. 2010, 31, 1427.

[64] Tumula, V. R.; Bondwal, S.; Bisht, P.; Penden, C.; Kumar, J. React. Kinet., Mech. Catal. 2012, 107, 449.

[65] Lindén, A. A.; Krüger, L.; Bäckvall, J.-E. J. Org. Chem. 2003, 68, 5890.

[66] Lindén, A. A.; Johansson, M.; Hermanns, N.; Bäckvall, J.-E. J. Org. Chem. 2006, 71, 3849 .
[67] Marsh, B. J.; Carbery, D. R. Tetrahedron Lett. 2010, 51, 2362

[68] Bakavoli, M.; Kakhky, A. M.; Shiri, A.; Ghabdian, M.; Davoodnia, A.; Eshghi, H.; Khatami, M. Chin. Chem. Lett. 2010, 21, 651.

[69] Rostami, A.; Akradi, J. Tetrahedron Lett. 2010, 51, 3501.

[70] (a) Mojr, V.; Herzig, V.; Buděšínský, M.; Cibulka, R.; Kraus, T. Chem. Commun. 2010, 46, 7599.

(b) Mojr, V.; Buděšínský, M.; Cibulka, R.; Kraus, T. Org. Biomol. Chem. 2011, 9, 7318 .

(c) Hartman, T.; Herzig, V.; Buděšínský, M.; Jindřich, J.; Cibulka, R.; Kraus, T. Tetrahedron: Asymmetry 2012, 23, 1571.

[71] Huang, Y. B.; Yi, W. B.; Cai, C. J. Fluorine Chem. 2011, 132, 554.

[72] Liu, Z.-M.; Zhao, H.; Li, M.-Q.; Lan, Y.-B.; Yao, Q.-B.; Tao, J.-C.; Wang, X.-W. Adv. Synth. Catal. 2012, 354, 1012

[73] Liao, S.; Čorić, I.; Wang, Q.; List, B. J. Am. Chem. Soc. 2012, 134, 10765.

[74] Iida, H.; Iwahana, S.; Mizoguchi, T.; Yashima, E. J. Am. Chem. Soc. 2012, 134, 15103.

(Li, L.) 